ENTREVISTAS

Rev Chil Salud Pública 2013. Vol 17 (2): 147-150

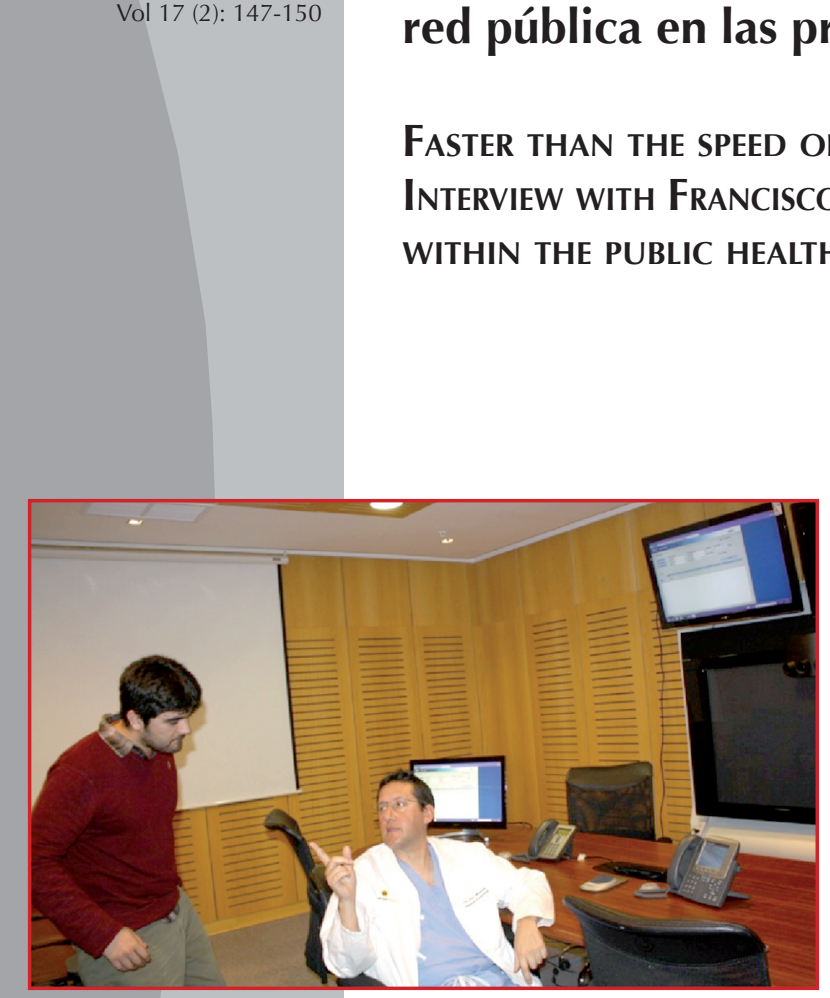

\section{Francisco Albornoz o moviendo la prodigiosa red pública en las proximidades de $\mathrm{C}$}

\author{
FASTER THAN THE SPEED OF LIGHT: \\ AlborNOZ ON THE USE OF TELEMEDICINE \\ HCARE NETWORK IN C
}

En el año 2000, en medio del debate sobre la reforma, Bitrán y Almarza enumeraban como incentivos de los médicos para sobreprescribir prestaciones "[...] comodidad y seguridad: por ejemplo, con un mayor número de exámenes es más segura y descansada la labor del médico, aunque estos no tengan un alto rendimiento de diagnóstico; se sustituye la labor médica por otras prestaciones".

Trece años después de publicada esta sentencia categórica acerca de las tecnologías y el trabajo médico, que enjuicia la actividad médica como un cansancio y a las tecnologías como un tercero en quien se descarga la labor, tenemos la fortuna de conocer la experiencia de un equipo de profesionales y técnicos, que mueven cielo y tierra porque consideran que sus pacientes son pocos. $Y$ con ese aliento en el corazón, promueven un sistema de telemedicina que revoluciona el funcionamiento de la referencia, la contra-referencia y la especialidad misma. Partiendo de un sistema de telemedicina para interconsultas cardiológicas entre el Hospital Las Higueras y los hospitales de San Carlos y Curanilahue, ba devenido una red de diagnóstico y manejo que articula cerca de 20 centros de atención. La red actualmente se extiende a cardiología, cirugia cardiaca, psiquiatría, nefrología y ya empieza a viralizar a la medicina interna y subespecialidades. Todo esto en Talcahuano, en menos de 5 años, con

Yuri Carvajal, INGRID GONZÁLEZ ${ }^{2}$, Jorge Pacheco ${ }^{3}$

'Investigador Fondecyt Controversias tecno-científica en la reforma de salud: análisis desde la sociología de la traducción Proyecto id 3130585,

Escuela de Salud Pública, Universidad de Chile ycarvajal@med.uchile.cl

${ }^{2}$ Escuela de Enfermería,

Universidad de Concepción gringrind6@gmail.com

${ }^{3}$ Escuela de Sociología,

Universidad de Concepción jorge.pacheco260@gmail.com

Yuri Carvajal agradece el apoyo del Fondecyt al proyecto 3130585 estatuto administrativo, Chilecompra, terremoto y alta dirección. Y además, con un Ministerio de Salud que atiende, ya sabemos, en Mac-Iver 541.

Viajamos al Hospital Higueras para conocer algo acerca de cómo se inician las innovaciones tecnológicas en salud. Abi entrevistamos al Dr. Francisco Albornoz quien ha liderado este proyecto desde su regreso a Chile en el 2004 tras formarse como cardiólogo intervencionista en Estados Unidos. Nuestro primer encuentro fue reconocerlo como uno de los tantos Dédalos de la medicina chilena, que han resuelto enigmas tecnológicos, desenredado laberintos técnicos, remontado dificultades objetuales, capacitado con nuevas re-descripciones de antiguos problemas: un convencido introductor de tecnologías médicas, otro nuevo hombre de las redes tecno-científicas en la salud, que han descubierto desde la clínica y la salud pública, el amor por las técnicas.

La siguiente transcripción es una versión parcial de lo conversado el 24 de mayo en el Hospital Higueras.

Ricardo Bitrán y Francisco Xavier Almarza Las Instituciones de Salud Previsional (ISAPRES) en Chile 463-570 en Daniel Titelman/Andras Uthoff Compiladores Ensayos Sobre el Financiamiento de la Seguridad Social Los casos de Estados Unidos-Canadá Argentina -Chile-Colombia FCE Santiago 2000 Volumen II, p. 508. 
Dr. Albornoz: Como te contaba, nosotros comenzamos hace aproximadamente 6 años, de hecho en marzo de 2008 lanzamos la primera iniciativa. Cuando quieres meterte en el tema de las tecnologías de la información y la comunicación aplicadas a la salud, te encuentras con una cosa un poco difícil en el sentido de que hay muchas variables, el primer problema está asociado a la definición de qué es un acto médico versus un acto tele-médico, porque en dicha definición hay implicancias no solo tecnológicas que tienen que ver con el componente tele, sino además éticas, administrativas, económicas y técnicas, es decir, qué es lo que debe este acto llevar adentro, la privacidad, la seguridad. Qué es lo que omites en un acto telemédico, qué es probablemente importante para la toma de una decisión racional y certera, etc, etc. De modo que no era sencillo.

Si bien es cierto que nuestro país tiene mucho desarrollo en el uso de las TIC's en la banca, en medicina la cosa es más compleja, por eso creo que vamos más atrás. Aun cuando Chile, lidera la disponibilidad de banda ancha en el contexto latinoamericano, todavía estamos muy atrasados en la incorporación de las TIC's a la salud. Los esfuerzos que se han estado haciendo, los cuales son lógicos, están vinculados a la incorporación de los HIS (Sistemas de Información Hospitalaria) a los grandes hospitales lo que ha sucedido en forma un tanto lenta y disímil. Sin embargo, hay evidencia importante en la experiencia americana y europea, que los HIS, pensados como soluciones intrahospitalarias para mejorar la administración hospitalaria no mejoran los problemas de acceso, oportunidad y equidad propios de un ecosistema de instituciones. Es en este ecosistema en donde tienen lugar los procesos tele-médicos. Por tanto el HIS y el acto tele-médico son cosas distintas. Nosotros decidimos usar las TICs para enfrentar los históricos problemas de acceso, oportunidad y equidad en nuestro ecosistema de instituciones sanitarias públicas interviniendo, en primer lugar en el nodo crítico de la referencia y la contra-referencia.

En base a esa lógica creamos un proyecto piloto. Con tecnología simple llevamos la interconsulta manuscrita a una aplicación web que permitía una interacción asincrónica entre un médico general en un establecimiento lejano y un médico especialista en otro establecimiento. Pero redefini- mos dicha interconsulta como un proceso de cuidado continuo, gatillado por la descripción de un problema clínico con una historia y examen físico, que necesita una primera respuesta elaborada y razonada por el especialista a dicho problema y que gatilla un estado intermedio de exámenes (invasivos y no invasivos), los cuales una vez obtenidos generan una segunda respuesta del especialista que cierra el ciclo diagnóstico con una propuesta terapéutica y un plan de seguimiento. Como te das cuenta esto no es meramente la solicitud de una hora en un formulario manuscrito, sino que ahora había una estructura con interacción de información y conocimiento (o sea referencia y contrareferencia) entre colegas tratando de resolver un problema a un paciente.

Con esto, redefinimos el concepto de interconsulta en el 2008. El impacto de esa re-definición, y eso no es tecnología, es proceso, fue que adaptamos la tecnología a nuestro proceso y lo que logramos fue dramático. Antes del modelo en los dos lugares que intervenimos, el hospital de Curanilahue y el hospital de San Carlos con médicos generales, nos encontramos con que la primera respuesta del especialista en el modelo tradicional o presencial no ocurría antes de un año versus un promedio de 54 horas en el nuevo modelo telemédico. Y la solución intervencionista o farmacológica de dichos casos, caía de dos años en el modelo tradicional a tres o cuatro semanas en el modelo telemédico. Desaparecieron las listas de espera no por cosmética sino por oportunidad y eficiencia real. Y los médicos que participaban en esto, principalmente los médicos generales, empezaron a aprender medicina cardiovascular, al punto que un año después su nivel de conocimiento y manejo, basado en esta educación continua que el especialista hacía, en términos de discutir el diagnostico diferencial de síncope, angina, etc. su nivel de conocimiento se empezó a ir hacia arriba. Eso nos encendió las luces y nos permitió seguir escalando en complejización tecnológica, y en ir agregando nuevos elementos a esta definición inicial y le agregamos electrocardiografía en línea, test de esfuerzo en línea, eco-cardiografía en línea e interacción con video en tiempo real, de muy alta calidad, todo bajo estándares que consideramos son los que debe tenerse para hacer actos tele-médicos. 
Los datos preliminares de cuatro años de trabajo los socializamos dentro de los tomadores de decisión tanto a nivel de la macro-regional Biobío, que son cinco Servicios de Salud, como a nivel de la dirección general de redes del Ministerio de salud, y eso implicó, que nos ganáramos un grant de un millón de dólares para hacer crecer este piloto, y transformarlo en un modelo operacional escalable. Eso es lo que a fin de mes inauguramos, un ecosistema de instituciones públicas representadas en veinte sitios y un centro de especialidad funcionando bajo una tele-red que incorpora todos estos elementos adentro, más un engranaje continuo de especialidades que se empiezan a subir al modelo.

Hemos aprendido que, primero, la tecnología es importante, o sea no puedes hacerlo con cualquier cosa, hay estándares. Segundo, la tecnología no es suficiente, el motor de este asunto está asociado a la definición de los procesos y los nodos clínicos que tu quieres intervenir, tienen que tener sentido y engranarse dentro de la lógica sanitaria pública chilena, o sea no puedes hacer cosas que no están dentro de lo que se considera lo normal que ocurra dentro del tráfico de pacientes y el registro de prestaciones en Chile. Tercero, los usuarios finales, esto es el equipo de salud que provee directamente el servicio o prestación clínica deben definir el flujo de trabajo. Es un error tratar de imponer flujos de trabajo creados en un escritorio por personas que no ven pacientes o no manejan la especialidad.

Cuarto, a nivel administrativo tuvimos que reprogramar los horarios de los especialistas. Hoy día tenemos especialistas en este hospital, que tienen un contrato por horas presenciales, $y$ va a su policlínico o a su laboratorio a hacer lo que hacen los doctores, pero también tiene un policlínico virtual y va a la sala de tele-presencia y entrevista pacientes en tiempo real viajando de ciudad en ciudad apretando un botón, responde interconsultas en línea, informa electrocardiogramas, test de esfuerzo y ecocardiogramas en la sala de telepresencia, desde su casa o en donde se encuentre. Este especialista tiene una "canasta virtual" de pacientes y exámenes de los cuales es responsable independiente de su presencia física en el hospital. A su vez, el médico general ahora cuenta con una "nube" de especialistas que lo asisten en el cuidado de su paciente. Como te das cuenta giramos a un modelo basado en "servicios centrados en la institución" a uno de "servicios centrados en el paciente".

Quinto, cambiamos roles tradicionales y dimos un paso más entrenando enfermeras y hoy día, estas enfermeras están haciendo ecografías cardíacas. Todas las ecografías se van a la nube y el cardiólogo las informa. Estamos a un rendimiento de 10 minutos por tele informe de ecografía cardíaca, y no 35 o 40 minutos por ecocardiograma presencial. Inyectamos hora/ cardiólogo al sistema al sacarle tareas físicas al cardiólogo, que pueden ser hechas por otro profesional entrenado, entonces todo eso desde el punto de vista sanitario, para el tomador de decisión, para el administrador, hace mucho sentido.

Sexto, hemos generado también un modelo de contra-referencia para los médicos generales de la atención primaria no sólo por las respuestas que damos en las interconsultas, sino que además por la participación en las reuniones clínicas, que ya no es una reunión entre nosotros, sino que se suben a la reunión desde los sitios remotos y eso es vital, porque a veces se nos olvida lo que es trabajar en un consultorio o trabajar en un hospital general. Fui médico general a mucha honra y estás solo. Por lo tanto esta cosa de tener acceso todas las semanas y participar del conocimiento genera entusiasmo y educación continua. La percepción es que tu hospital creció y ahora eres parte de una tele-red. El paso siguiente que ya lo estamos haciendo es ampliarnos a un "cloud de cooperación internacional" con un centro en España que quiere trabajar con nosotros y otro en Estados Unidos.

\section{¿También es público el de España?}

El de España es público, el de Estados Unidos es privado, que es la universidad donde me formé. Eso lo vamos a formar ahora teniendo la nube arriba, porque para todo esto se necesita una nube de administración de video profesional.

Generamos además dentro de la empresa privada, un interés por desarrollar el área. En este caso debo destacar, y no tengo ninguna relación de nada, pero voy a destacar lo que está haciendo Telefónica, ellos construyeron un cloud de video dedicado, que inter-opera con distintas tecnologías propietarias y permite conectividad con Europa y con Estados Unidos. Eso no estaba en 
el país, te fijas, entonces comienza la innovación transformacional.

\section{¿Y todo alrededor de esto?}

Alrededor de esto. Ahora hemos iniciado diálogo con REUNA, justamente por el mismo tema. Yo diría que lo que está ocurriendo no es una innovación, es una revolución. La intención nuestra es estandarizar, sistematizar, y hacer que los distintos participantes del tele-ecosistema: los tomadores de decisión, los proveedores de servicios tecnológicos, los creadores de tecnología, la academia, los pagadores de los servicios, (FONASA, ISAPRES), los servicios de salud y hospitales, las municipalidades y los usuarios finales (equipo de salud y pacientes) participen de esta transformación. Anticipamos que el mapa sanitario público va a cambiar y cambiará muy rápido.

Tenemos una unidad de teleprocesos que depende de la subdirección médica, que si bien es cierto nació en cardiología, no pretende quedarse en cardiología. Hoy día ya tenemos a los psiquiatras arriba, los nefrólogos se están subiendo, los neurólogos vienen ahí en camino y queremos subir a los internistas, los pediatras, o sea, esto es aplicable a toda la medicina. El desafío es cómo lo complementas con la medicina presencial con su paradigma tradicional.

Esa complementariedad tiene que estar pensada para intervenir los nodos críticos que hoy día hacen que nuestro sistema sea como es y con las limitaciones que tiene. A nuestro juicio el nodo crítico más importante es la referencia y la contra-referencia, o sea el acceso oportuno. No es normal que alguien se muera o secuele esperando una hora, eso no es normal. Pero el sistema de referencia y contra referencia tradicional es ciego. ¿Quién recibe la interconsulta? ¿Cuál es su prioridad ?, y con una letra infame cuando es manuscrita. La interconsulta tiene que ir de médico general a especialista directamente y en forma instantánea. Si yo estoy en un consultorio y tengo un paciente que necesita nefrólogo, neurólogo y cardiólogo, esa interconsulta se tiene que direccionar directa- mente a esos especialistas, no a un intermediario, que lo único que hace es burocratizar el proceso clínico. Estos han sido los nodos que hemos estado interviniendo con toda esta definición. No ha sido la choreza de ver las pantallas y la tecnología que tenemos, que es maravillosa. Esto es una consecuencia, el motor está asociado a esto otro.

En estas innovaciones se conjugan varios factores, que viniste, que te formaste en Estados Unidos, que te gustaba la tecnología, pero también puede ser que algunas cosas de cardiología no estaban siendo resueltas.

Sin duda, esto nació de mi interés por resolver problemas. Yo llegué en el 2004, me traje una donación de Estados Unidos para crear un laboratorio de medicina intervencionista que es lo que yo hago. Lo hicimos, un millón de dólares, un nuevo laboratorio, etc. Pero todavía no aterrizaba, y empecé a participar de las reuniones de la macro regional, no entendía cómo funcionaba la red pública sanitaria. Me empiezo a enterar de cómo funciona en términos reales, que me parece lógicamente organizada, pero con problemas de gestión. Cuando empecé a escuchar el problema que tenía el director del Hospital de San Carlos o el del CESFAM de Talcahuano, con sus listas de espera con sus planes y problemas no resueltos para los enfermos, con el problema de traslado de los enfermos, con los pacientes intervenidos que volvían y que nadie sabía qué hacer con ellos, ¿te fijas?. Y ahí surgió: ¿cómo vamos a enfrentar este problema? Iniciativas hay muchas, desde entrenar más gente, todo lo que tú quieras, pero dijimos vamos a crear una propuesta incorporando las TIC's. Ese fue el tema, al menos eso fue lo que yo vi, ahora el desafío fue, en qué, cómo las uso, porque las TIC's no son terapia. Son una herramienta. Creo que ahí estuvo la inteligencia, el resto ha sido crecer y crecer y vamos a ver muchas más novedades. El interés nuestro ha sido aquí crear una especie de laboratorio viviente, un open living lab, para que la data que aquí se genere, y el conocimiento que podamos adquirir sea escalable y replicable en el resto del país, sin duda. 